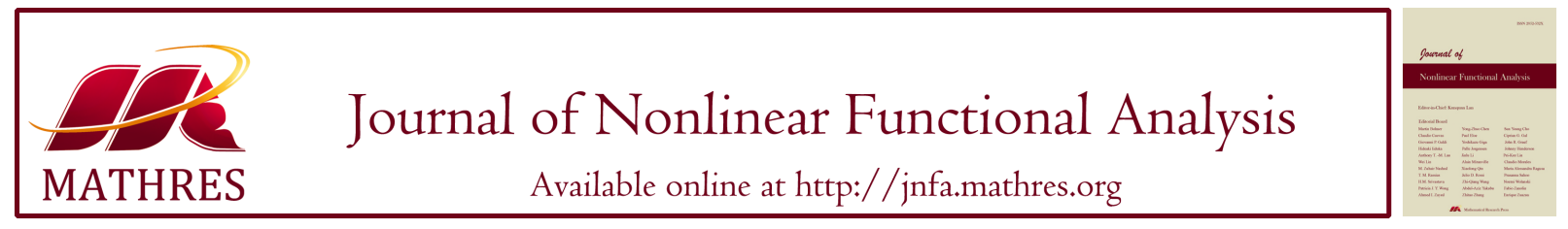

\title{
ON A THREE SPECIES RATIO-DEPENDENT LOTKA-VOLTERRA COOPERATIVE SYSTEM WITH DELAYS
}

\author{
GULIBAIKEREMU ABULIMITI ${ }^{1}$, AHMADJAN MUHAMMADHAJI ${ }^{1, *}$, ROUZIMAIMAITI MAHEMUTI $^{1,2}$, \\ AZHAR HALIK ${ }^{1}$ \\ ${ }^{1}$ College of Mathematics and System Sciences, Xinjiang University, Urumqi 830046, China \\ ${ }^{2}$ Guangdong Provincial Key Laboratory of Computational Science and Material Design, \\ Southern University of Science and Technology, Shenzhen, China
}

\begin{abstract}
A class of non-autonomous three species Lotka-Volterra cooperative system with ratio-dependent functional responses and delays is discussed. A set of easily verifiable new sufficient conditions on the permanence, the existence of positive periodic solutions, and the global attractivity of the system are established by using the comparison method, and the construction of Lyapunov functions. Finally, a numerical simulation is given to verify the effectiveness of the obtained results.
\end{abstract}

Keywords. Ratio-dependent cooperative system; Permanence; Periodic solution; Time delay; Global attractivity.

\section{INTRODUCTION}

In the past few years, the study of population dynamical systems with ratio-dependent functional responses has become a main concern for researchers. Especially, a lot of authors investigated the population predator-prey system with ratio-dependent functional responses [1]-[6]. In these studies, predators were considered as a ratio-dependent functional response to describe the interactions between predators and prey. For example, Muhammadhaji and Teng [6] proposed and discussed the following non-delayed competing predator-prey system with ratio-dependent

${ }^{*}$ Correspoinding author.

E-mail address: ahmatjanam@aliyun.com (A. Muhammadhaji).

Received March 4, 2021; Accepted May 10, 2021.

(C)2021 Journal of Nonlinear Functional Analysis 
functional responses and stage structure

$$
\begin{aligned}
\dot{x}_{1}(t)= & r(t) x_{2}(t)-B(t) x_{1}(t)-d_{1}(t) x_{1}^{2}(t), \\
\dot{x}_{2}(t)= & B(t) x_{1}(t)-d_{2}(t) x_{2}^{2}(t)-\frac{a_{1}(t) x_{2}(t) y_{1}(t)}{k(t) x_{2}^{2}(t)+\beta_{1}(t) x_{2}(t)+\alpha_{1}(t)} \\
& -\frac{a_{2}(t) x_{2}(t) y_{2}(t)}{\alpha_{2}(t)+\beta_{2}(t) x_{2}(t)+\gamma(t) y_{2}(t)}, \\
\dot{y}_{1}(t)= & y_{1}(t)\left(-d_{3}(t)+\frac{e_{1}(t) x_{2}(t)}{k(t) x_{2}^{2}(t)+\beta_{1}(t) x_{2}(t)+\alpha_{1}(t)}-D_{1}(t) y_{1}(t)-\frac{c_{1}(t) y_{2}(t)}{b_{1}(t)+y_{2}(t)}\right), \\
\dot{y}_{2}(t)= & y_{2}(t)\left(-d_{4}(t)+\frac{e_{2}(t) x_{2}(t)}{\alpha_{2}(t)+\beta_{2}(t) x_{2}(t)+\gamma(t) y_{2}(t)}-D_{2}(t) y_{2}(t)-\frac{c_{2}(t) y_{1}(t)}{b_{2}(t)+y_{1}(t)}\right),
\end{aligned}
$$

where $x_{1}(t)$ represents the density of immature individuals preys at time $t, x_{2}(t)$ represents the density of mature individuals preys at time $t, y_{1}(t)$ and $y_{2}(t)$ represent the density of two competing predators at time $t$, respectively. In system (1.1), Muhammadhaji and Teng employed the Holling and Beddington-DeAngelis type functional response to describe the interactions between predators and prey.

It is worth noting that, after the pioneering work of May [7], some of studies on the population dynamical systems has been carried out on the cooperative model with ratio-dependent functional response [7]-[15], namely, May cooperative model. For example, Muhammadhaji, Teng and Abdurahman [11] studied the following non-autonomous stage-structured cooperative system with ratio-dependent functional response and delay

$$
\begin{aligned}
& \dot{x}_{1}(t)=r_{1}(t) x_{2}(t)-d_{1}(t) x_{1}(t)-r_{1}(t-\tau) e^{-\int_{t-\tau}^{t} d_{1}(s) d s} x_{2}(t-\tau), \\
& \dot{x}_{2}(t)=r_{1}(t-\tau) e^{-\int_{t-\tau}^{t} d_{1}(s) d s} x_{2}(t-\tau)-d_{2}(t) x_{2}^{2}(t)-\frac{c_{1}(t) x_{2}(t)}{a_{1}(t)+b_{1}(t) y(t)}, \\
& \dot{y}(t)=y(t)\left[r_{2}(t)-d(t) y(t)-\frac{c_{2}(t)}{a_{2}(t)+b_{2}(t) x_{2}(t)}\right] .
\end{aligned}
$$

In system (1.2), Muhammadhaji, Teng and Abdurahman used $\frac{c_{1}(t) x_{2}(t)}{a_{1}(t)+b_{1}(t) y(t)}$, and $\frac{c_{2}(t)}{a_{2}(t)+b_{2}(t) x_{2}(t)}$ to describe the cooperative relations between species $x$ and $y$. By using the comparison method, the sufficient conditions on the boundedness, permanence and extinction were established for system (1.2). In [14], Yang et al. considered the following three species non-autonomous Lotka-Volterra predator-prey-mutualist systems with ratio-dependent functional response

$$
\begin{aligned}
& \dot{x}(t)=x(t)\left[a_{1}(t)-b_{1}(t) x(t)-\frac{c_{1}(t) z(t)}{d_{1}(t)+d_{2}(t) y(t)}\right], \\
& \dot{y}(t)=y(t)\left[a_{2}(t)-\frac{y(t)}{d_{3}(t)+d_{4}(t) x(t)}\right] \\
& \dot{z}(t)=z(t)\left[-a_{3}(t)+\frac{c_{2}(t) x(t)}{d_{1}(t)+d_{2}(t) z(t)}\right] .
\end{aligned}
$$

In system (1.3), Yang et al. used

$$
\frac{c_{1}(t)}{d_{1}(t)+d_{2}(t) y(t)}
$$




$$
\frac{y(t)}{d_{3}(t)+d_{4}(t) x(t)},
$$

and

$$
\frac{c_{2}(t) x(t)}{d_{1}(t)+d_{2}(t) z(t)}
$$

to describe the predator, prey and cooperative relations between species $x, y$ and $z$. And they obtained some sufficient conditions for the permanence and periodic solution of system (1.3) by employing the comparison method.

However, most of the studies on the Lotka-Volterra systems with ratio-dependent functional response mainly concerned with the permanence, extinction and the existence of positive periodic solutions [11]-[15]. As the global attractivity of the Lotka-volterra cooperative systems with ratio-dependent functional response have not been fully investigated, in this paper, we consider the following non-autonomous three species Lotka-Volterra cooperative system with ratio-dependent functional responses and delays

$$
\begin{aligned}
& \dot{y}_{1}(t)=y_{1}(t)\left[r_{1}(t)-a_{11}(t) y_{1}(t)-\frac{b_{1}(t) y_{1}(t-\tau)}{a_{12}(t) y_{2}(t-\tau)+a_{13}(t) y_{3}(t-\tau)+c_{1}(t)}\right], \\
& \dot{y}_{2}(t)=y_{2}(t)\left[r_{2}(t)-a_{22}(t) y_{2}(t)-\frac{b_{2}(t) y_{2}(t-\tau)}{a_{21}(t) y_{1}(t-\tau)+a_{23}(t) y_{3}(t-\tau)+c_{2}(t)}\right], \\
& \dot{y}_{3}(t)=y_{3}(t)\left[r_{3}(t)-a_{33}(t) y_{3}(t)-\frac{b_{3}(t) y_{3}(t-\tau)}{a_{31}(t) y_{1}(t-\tau)+a_{32}(t) y_{2}(t-\tau)+c_{3}(t)}\right] .
\end{aligned}
$$

Our main purpose is to establish some new sufficient conditions on the permanence, existence of positive periodic solution and global attractivity of system (1.4). The method used in this paper is the comparison method and the Lyapunov function method.

\section{PRELIMINARIES}

In system (1.4), $y_{i}(t)(i=1,2,3)$ represent the density of three species $y_{i}(i=1,2,3)$ at time $t$, respectively; $r_{i}(t)(i=1,2,3)$ represent the intrinsic growth rate of three species $y_{i}(i=1,2,3)$ at time $t$, respectively; $a_{i i}(t)(i=1,2,3)$ represent the intrapatch restriction density of three species $y_{i}(i=1,2,3)$ at time $t$, respectively; $a_{i j}(t)(i \neq j, i, j=1,2,3)$ represent the cooperative coefficients between three species $y_{i}(i=1,2,3)$ at time $t$, respectively.

$$
\begin{gathered}
\frac{b_{1}(t) y_{1}(t-\tau)}{a_{12}(t) y_{2}(t-\tau)+a_{13}(t) y_{3}(t-\tau)+c_{1}(t)}, \\
\frac{b_{2}(t) y_{2}(t-\tau)}{a_{21}(t) y_{1}(t-\tau)+a_{23}(t) y_{3}(t-\tau)+c_{2}(t)},
\end{gathered}
$$

and

$$
\frac{b_{3}(t) y_{3}(t-\tau)}{a_{31}(t) y_{1}(t-\tau)+a_{32}(t) y_{2}(t-\tau)+c_{3}(t)}
$$

describe the cooperative relations between species $y_{1}, y_{2}$, and $y_{3} . \tau$ is a positive constant.

In this paper, the initial conditions for system (1.4) take the following form

$$
y_{i}(t)=\phi_{i}(t) \text { for all } t \in[-\tau, 0], i=1,2,3,
$$

where $\phi_{i}(t)(i=1,2,3)$ are nonnegative continuous functions defined on $[-\tau, 0]$ satisfying $\phi_{i}(0)>0(i=1,2,3)$. 
Throughout this paper, for system (1.4), we introduce the following hypotheses.

$\left(H_{1}\right) \quad r_{i}(t), a_{i i}(t), c_{i}(t)(i=1,2,3)$ and $a_{i j}(t)(i \neq j, i, j=1,2,3)$ are continuous, bounded and strictly positive functions on $[0,+\infty)$.

$\left(H_{2}\right) \quad r_{i}(t), a_{i i}(t), c_{i}(t)(i=1,2,3)$ and $a_{i j}(t)(i \neq j, i, j=1,2,3)$ are all continuously positive $\omega$-periodic functions on $[0, \omega]$.

Throughout this paper, for any continuous function $f(t)$, we denote

$$
f^{L}=\inf _{t \in[0,+\infty)} f(t), f^{M}=\sup _{t \in[0,+\infty)} f(t) .
$$

Now, we present some useful definition and lemmas.

Definition 2.1. System (1.4) is said to be global attractive if, for any two positive solutions $\left(y_{1}(t), y_{2}(t), y_{3}(t)\right)$ and $\left(x_{1}(t), x_{2}(t), x_{3}(t)\right)$ of system $(1.4)$, then

$$
\lim _{t \rightarrow \infty}\left(x_{i}(t)-y_{i}(t)\right)=0, \quad i=1,2,3 .
$$

Lemma 2.2. [6] Consider the following equation: $\dot{u}(t)=u(t)\left(d_{1}-d_{2} u(t)\right)$, where $d_{2}>0$.

(1) If $d_{1}>0$, then $\lim _{t \rightarrow+\infty} u(t)=d_{1} / d_{2}$.

(2) If $d_{1}<0$, then $\lim _{t \rightarrow+\infty} u(t)=0$.

Lemma 2.3. [16] Assume that, for $y(t) \geq 0$,

$$
\dot{y}(t) \geq y(t)\left(\lambda-\sum_{l=0}^{m} \mu^{l} y(t-l \tau)\right) .
$$

Then, there exists a positive constant $m_{y}>0$ such that, for $\mu=\sum_{l=0}^{m} \mu^{l}>0$,

$$
\liminf _{t \rightarrow \infty} y(t) \geq m_{y}=\frac{\lambda}{\mu} \exp \left\{\left(\lambda-\mu M_{y}\right) m \tau\right\}>0,
$$

where $\lim \sup _{t \rightarrow \infty} y(t) \leq M_{y}<+\infty$.

Lemma 2.4. [17] If there exist positive constants $m$ and $M$ for any $\Phi \in C_{+}^{n}[-\tau, 0]$ such that

$$
m<\liminf _{t \rightarrow \infty} x_{i}(t, 0, \Phi) \leq \limsup _{t \rightarrow \infty} x_{i}(t, 0, \Phi)<M, \quad i=1,2, \cdots, n .
$$

then the following periodic differential equation

$$
\frac{d x}{d t}=F\left(t, x_{t}\right)
$$

admits at least one positive $\omega$-periodic solution, where $F\left(t, x_{t}\right)$ is a n-dimensional continuous functional, $x(t) \in R^{n}$ and $x(t, 0, \Phi)=\left(x_{1}(t, 0, \Phi), x_{2}(t, 0, \Phi), \cdots, x_{n}(t, 0, \Phi)\right)$ is a solution of the functional differential equation with initial condition $x_{0}=\Phi$.

\section{Main Results}

Theorem 3.1. If $\left(H_{1}\right)$ holds, then system (1.4) is permanent.

Proof. Let $y(t)=\left(y_{1}(t), y_{2}(t), y_{3}(t)\right)$ be a any positive solution to system (1.4) with initial condition (2.1). From system (1.4), for $t \geq \tau$, we have

$$
\dot{y}_{i}(t) \leq y_{i}(t)\left[r_{i}(t)-a_{i i}(t) y_{i}(t)\right], \quad i=1,2,3 .
$$


From Lemma 2.2 and the comparison theorem, there exists a constant $T_{1}>0$ such that

$$
y_{i}(t) \leq \frac{r_{i}^{M}}{a_{i i}^{L}}=M_{i}, \quad \text { as } t>T_{1}, i=1,2,3 .
$$

Next, using system (1.4) again, for $t \geq \tau$, we have

$$
\dot{y}_{i}(t) \geq y_{i}(t)\left[r_{i}(t)-a_{i i}(t) y_{i}(t)-b_{i}(t) y_{i}(t-\tau)\right], \quad i=1,2,3 .
$$

From Lemma 2.4, there exists a constant $T_{2}>T_{1}$ such that

$$
y_{i}(t) \geq \frac{r_{i}^{L}}{a_{i i}^{M}+b_{i}^{M}} \exp \left\{\left[r_{i}^{L}-\left(a_{i i}^{M}+b_{i}^{M}\right) M_{i}\right] \tau\right\}=m_{i}, \quad \text { as } t>T_{2}, i=1,2,3 .
$$

This completes the proof of this theorem.

Remark 3.2. From the proof of Theorem 3.1, we can see that if species $y_{1}(t), y_{2}(t)$, and $y_{3}(t)$ have a positive intrinsic growth rate, then system (1.4) must be permanent.

As a direct result of Lemma 2.4, we have from Theorem 3.1 the following result.

Corollary 3.3. Assume that $\left(\mathrm{H}_{2}\right)$ holds. Then, system (1.4) is permanent and admits at least one positive $\omega$-periodic solution.

Now, we will obtain the sufficient conditions for the global attractivity of system (1.4). First, we denote

$$
\begin{gathered}
A_{1}(t)=\frac{b_{1}(t) c_{1}(t)}{\Gamma_{1}(t)} \leq \frac{b_{1}^{M} c_{1}^{M}}{\Gamma_{1}^{L}}=A_{1}^{M}, \\
A_{2}(t)=\frac{b_{1}(t) a_{12}(t) x_{2}(t-\tau)}{\Gamma_{1}(t)} \leq \frac{b_{1}^{M} a_{12}^{M} M_{2}}{\Gamma_{1}^{L}}=A_{2}^{M}, \\
A_{3}(t)=\frac{b_{1}(t) a_{13}(t) x_{3}(t-\tau)}{\Gamma_{1}(t)} \leq \frac{b_{1}^{M} a_{13}^{M} M_{3}}{\Gamma_{1}^{L}}=A_{3}^{M}, \\
A_{4}(t)=\frac{b_{1}(t) a_{12}(t) x_{1}(t-\tau)}{\Gamma_{1}(t)} \leq \frac{b_{1}^{M} a_{12}^{M} M_{1}}{\Gamma_{1}^{L}}=A_{4}^{M}, \\
A_{5}(t)=\frac{b_{1}(t) a_{13}(t) x_{1}(t-\tau)}{\Gamma_{1}(t)} \leq \frac{b_{1}^{M} a_{13}^{M} M_{1}}{\Gamma_{1}^{L}}=A_{5}^{M}, \\
A_{6}(t)=\frac{b_{2}(t) c_{2}(t)}{\Gamma_{2}(t)} \leq \frac{b_{2}^{M} c_{2}^{M}}{\Gamma_{2}^{L}}=A_{6}^{M}, \\
A_{7}(t)=\frac{b_{2}(t) a_{21}(t) x_{1}(t-\tau)}{\Gamma_{2}(t)} \leq \frac{b_{2}^{M} a_{21}^{M} M_{1}}{\Gamma_{2}^{L}}=A_{7}^{M}, \\
A_{8}(t)=\frac{b_{2}(t) a_{23}(t) x_{3}(t-\tau)}{\Gamma_{2}(t)} \leq \frac{b_{2}^{M} a_{23}^{M} M_{3}}{\Gamma_{2}^{L}}=A_{8}^{M}, \\
A_{10}(t)=\frac{b_{2}(t) a_{23}(t) x_{2}(t-\tau)}{\Gamma_{2}(t)} \leq \frac{b_{2}^{M} a_{21}^{M} M_{2}}{\Gamma_{2}^{L}}=A_{9}^{M}, \\
b_{2}(t) a_{21}(t) x_{2}(t-\tau)
\end{gathered}
$$




$$
\begin{gathered}
A_{11}(t)=\frac{b_{3}(t) c_{3}(t)}{\Gamma_{3}(t)} \leq \frac{b_{3}^{M} c_{3}^{M}}{\Gamma_{3}^{L}}=A_{11}^{M}, \\
A_{12}(t)=\frac{b_{3}(t) a_{31}(t) x_{1}(t-\tau)}{\Gamma_{3}(t)} \leq \frac{b_{3}^{M} a_{31}^{M} M_{1}}{\Gamma_{3}^{L}}=A_{12}^{M}, \\
A_{13}(t)=\frac{b_{3}(t) a_{32}(t) x_{2}(t-\tau)}{\Gamma_{3}(t)} \leq \frac{b_{3}^{M} a_{32}^{M} M_{2}}{\Gamma_{3}^{L}}=A_{13}^{M}, \\
A_{14}(t)=\frac{b_{3}(t) a_{31}(t) x_{3}(t-\tau)}{\Gamma_{3}(t)} \leq \frac{b_{3}^{M} a_{31}^{M} M_{3}}{\Gamma_{3}^{L}}=A_{14}^{M}, \\
A_{15}(t)=\frac{b_{3}(t) a_{32}(t) x_{3}(t-\tau)}{\Gamma_{3}(t)} \leq \frac{b_{3}^{M} a_{32}^{M} M_{3}}{\Gamma_{3}^{L}}=A_{15}^{M}, \\
B_{1}=a_{11}^{L}-\sum_{i=1}^{M} A_{i}^{M}-A_{9}^{M}-A_{14}^{M},
\end{gathered}
$$

and

$$
B_{2}=a_{22}^{L}-\sum_{i=6}^{8} A_{i}^{M}-A_{4}^{M}-A_{15}^{M}, \quad B_{3}=a_{33}^{L}-\sum_{i=10}^{13} A_{i}^{M}-A_{5}^{M}
$$

where

$$
\begin{gathered}
\Gamma_{1}(t)=\left(a_{12}(t) y_{2}(t-\tau)+a_{13}(t) y_{3}(t-\tau)+c_{1}(t)\right)\left(a_{12}(t) x_{2}(t-\tau)+a_{13}(t) x_{3}(t-\tau)+c_{1}(t)\right), \\
\Gamma_{2}(t)=\left(a_{21}(t) y_{1}(t-\tau)+a_{23}(t) y_{3}(t-\tau)+c_{2}(t)\right)\left(a_{21}(t) x_{1}(t-\tau)+a_{23}(t) x_{3}(t-\tau)+c_{2}(t)\right), \\
\Gamma_{3}(t)=\left(a_{31}(t) y_{1}(t-\tau)+a_{32}(t) y_{2}(t-\tau)+c_{3}(t)\right)\left(a_{31}(t) x_{1}(t-\tau)+a_{32}(t) x_{2}(t-\tau)+c_{3}(t)\right), \\
\Gamma_{1}^{L}=\left(a_{12}^{L} m_{2}+a_{13}^{L} m_{3}+c_{1}^{L}\right)^{2}, \\
\Gamma_{2}^{L}=\left(a_{21}^{L} m_{1}+a_{23}^{L} m_{3}+c_{2}^{L}\right)^{2},
\end{gathered}
$$

and

$$
\Gamma_{3}^{L}=\left(a_{31}^{L} m_{1}+a_{32}^{L} m_{2}+c_{3}^{L}\right)^{2} .
$$

Theorem 3.4. Suppose that $H_{1}$ holds and $B>0$. Then, system (1.4) is globally attractive, where $B=\min \left\{B_{1}, B_{2}, B_{3}\right\}$.

Proof. Suppose that $\left(y_{1}(t), y_{2}(t), y_{3}(t)\right)$, and $\left(x_{1}(t), x_{2}(t), x_{3}(t)\right)$ are any two positive solutions of system (1.4). From Theorem 3.1, there exist positive constants $M_{i}, m_{i}(i=1,2,3)$ and $T$ such that $m_{i} \leq y_{i}(t), x_{i}(t) \leq M_{i}(i=1,2,3)$ for all $t \geq T$. First, let $V_{1}(t)=\sum_{i=1}^{3}\left|\ln y_{i}(t)-\ln x_{i}(t)\right|$. 
Calculating the upper right derivative of $V_{1}(t)$ along system (1.4), we have

$$
\begin{aligned}
& D^{+} V_{1}(t)=\operatorname{sign}\left(y_{1}(t)-x_{1}(t)\right)\left[-a_{11}(t)\left(y_{1}(t)-x_{1}(t)\right)\right. \\
& -b_{1}(t)\left(\frac{y_{1}(t-\tau)}{a_{12}(t) y_{2}(t-\tau)+a_{13}(t) y_{3}(t-\tau)+c_{1}(t)}\right. \\
& \left.\left.-\frac{x_{1}(t-\tau)}{a_{12}(t) x_{2}(t-\tau)+a_{13}(t) x_{3}(t-\tau)+c_{1}(t)}\right)\right] \\
& +\operatorname{sign}\left(y_{2}(t)-x_{2}(t)\right)\left[-a_{22}(t)\left(y_{2}(t)-x_{2}(t)\right)\right. \\
& -b_{2}(t)\left(\frac{y_{2}(t-\tau)}{a_{21}(t) y_{1}(t-\tau)+a_{23}(t) y_{3}(t-\tau)+c_{2}(t)}\right. \\
& \left.\left.-\frac{x_{2}(t-\tau)}{a_{21}(t) x_{1}(t-\tau)+a_{23}(t) x_{3}(t-\tau)+c_{2}(t)}\right)\right] \\
& +\operatorname{sign}\left(y_{3}(t)-x_{3}(t)\right)\left[-a_{33}(t)\left(y_{3}(t)-x_{3}(t)\right)\right. \\
& -b_{3}(t)\left(\frac{y_{3}(t-\tau)}{a_{31}(t) y_{1}(t-\tau)+a_{32}(t) y_{2}(t-\tau)+c_{3}(t)}\right. \\
& \left.\left.-\frac{x_{3}(t-\tau)}{a_{31}(t) x_{1}(t-\tau)+a_{32}(t) x_{2}(t-\tau)+c_{3}(t)}\right)\right] \\
& =\operatorname{sign}\left(y_{1}(t)-x_{1}(t)\right)\left[-a_{11}(t)\left(y_{1}(t)-x_{1}(t)\right)+A_{4}(t)\left(y_{2}(t-\tau)-x_{2}(t-\tau)\right)\right. \\
& \left.+A_{5}(t)\left(y_{3}(t-\tau)-x_{3}(t-\tau)\right)-\sum_{i=1}^{3} A_{i}(t)\left(y_{1}(t-\tau)-x_{1}(t-\tau)\right)\right] \\
& +\operatorname{sign}\left(y_{2}(t)-x_{2}(t)\right)\left[-a_{22}(t)\left(y_{2}(t)-x_{2}(t)\right)+A_{9}(t)\left(y_{1}(t-\tau)-x_{1}(t-\tau)\right)\right. \\
& \left.+A_{10}(t)\left(y_{3}(t-\tau)-x_{3}(t-\tau)\right)-\sum_{i=6}^{8} A_{i}(t)\left(y_{2}(t-\tau)-x_{2}(t-\tau)\right)\right] \\
& +\operatorname{sign}\left(y_{3}(t)-x_{3}(t)\right)\left[-a_{33}(t)\left(y_{3}(t)-x_{3}(t)\right)+A_{14}(t)\left(y_{1}(t-\tau)-x_{1}(t-\tau)\right)\right. \\
& \left.+A_{15}(t)\left(y_{2}(t-\tau)-x_{2}(t-\tau)\right)-\sum_{i=11}^{13} A_{i}(t)\left(y_{3}(t-\tau)-x_{3}(t-\tau)\right)\right] \\
& \leq-\sum_{i=1}^{3} a_{i i}^{L}\left|y_{i}(t)-x_{i}(t)\right|+\left(\sum_{i=1}^{3} A_{i}^{M}+A_{9}^{M}+A_{14}^{M}\right)\left|y_{1}(t-\tau)-x_{1}(t-\tau)\right|+\left(\sum_{i=6}^{8} A_{i}^{M}\right. \\
& \left.+A_{4}^{M}+A_{15}^{M}\right)\left|y_{2}(t-\tau)-x_{2}(t-\tau)\right|+\left(\sum_{i=10}^{13} A_{i}^{M}+A_{5}^{M}\right)\left|y_{3}(t-\tau)-x_{3}(t-\tau)\right| .
\end{aligned}
$$

Next, we let

$$
\begin{aligned}
V_{2}(t)= & \left(\sum_{i=1}^{3} A_{i}^{M}+A_{9}^{M}+A_{14}^{M}\right) \int_{t-\tau}^{t}\left|y_{1}(s)-x_{1}(s)\right| d s \\
& +\left(\sum_{i=6}^{8} A_{i}^{M}+A_{4}^{M}+A_{15}^{M}\right) \int_{t-\tau}^{t}\left|y_{2}(s)-x_{2}(s)\right| d s+\left(\sum_{i=10}^{13} A_{i}^{M}+A_{5}^{M}\right) \int_{t-\tau}^{t}\left|y_{3}(s)-x_{3}(s)\right| d s .
\end{aligned}
$$


Calculating the upper right derivative of $V_{2}(t)$ and using (3.3), we have

$$
\begin{aligned}
D^{+} V_{1}(t)+D^{+} V_{2}(t) & \leq-B_{1}\left|y_{1}(t)-x_{1}(t)\right|-B_{2}\left|y_{2}(t)-x_{2}(t)\right|-B_{3}\left|y_{3}(t)-x_{3}(t)\right| \\
& \leq-\sum_{i=1}^{3} B\left|y_{i}(t)-x_{i}(t)\right| .
\end{aligned}
$$

Finally, we define a Lyapunov function as follows

$$
V(t)=V_{1}(t)+V_{2}(t)
$$

Calculating the upper right derivation of $V(t)$, from (3.5) we can obtain, for all $t \geq T$,

$$
D^{+} V(t) \leq-\sum_{i=1}^{3} B\left|y_{i}(t)-x_{i}(t)\right|
$$

Integrating from $T$ to $t$ on both sides of (3.6) produces

$$
V(t)+B \int_{T}^{t}\left(\sum_{i=1}^{3}\left|y_{i}(s)-x_{i}(s)\right|\right) d s \leq V(T)<+\infty .
$$

Hence, $V(t)$ bounded on $[T,+\infty)$, and we have

$$
\int_{T}^{t}\left(\sum_{i=1}^{3}\left|y_{i}(s)-x_{i}(s)\right|\right) d s \leq \frac{V(T)}{G}<+\infty
$$

which implies that

$$
\sum_{i=1}^{3}\left|y_{i}(t)-x_{i}(t)\right| \in L^{1}(T,+\infty)
$$

From the permanence of system (1.4), we can obtain that $\sum_{i=1}^{3}\left|y_{i}(t)-x_{i}(t)\right|$ is uniformly continuous on $[T,+\infty)$. By Barbalat's lemma, it follows that

$$
\lim _{t \rightarrow \infty}\left|y_{i}(t)-x_{i}(t)\right|=0,(i=1,2,3)
$$

This completes the proof of this theorem.

Corollary 3.5. Suppose that the conditions of Corollary 3.3 hold and B $>0$. Then, system (1.4) has a positive $\omega$ - periodic solution, which is globally attractive.

\section{NUMERICAL Simulation}

As an example, in this section, we consider the following Lotka-Volterra three species cooperative system with ratio-dependent functional responses and delays to illustrate the results 
obtained in this paper

$$
\begin{aligned}
\dot{y}_{1}(t)= & y_{1}(t)\left[3.95+0.65 \cos (t)-(4.15+0.25 \cos (t)) y_{1}(t)\right. \\
& \left.-\frac{(1.15+0.2 \cos (t)) y_{1}(t-0.25)}{(3+0.25 \cos (t)) y_{2}(t-0.25)+(3+0.2 \cos (t)) y_{3}(t-0.25)+2+0.25 \cos (t)}\right], \\
\dot{y}_{2}(t)= & y_{2}(t)\left[3.5+0.45 \cos (t)-(4.2+0.35 \cos (t)) y_{2}(t)\right. \\
& \left.-\frac{(1.2+0.15 \cos (t)) y_{2}(t-0.25)}{(3+0.2 \cos (t)) y_{1}(t-0.25)+(3+0.15 \cos (t)) y_{3}(t-0.25)+2+0.2 \cos (t)}\right], \\
\dot{y}_{3}(t)= & y_{3}(t)\left[3.25+0.35 \cos (t)-(4.1+0.55 \cos (t)) y_{3}(t)\right. \\
& \left.-\frac{(1.3+0.1 \cos (t)) y_{3}(t-0.25)}{(3.5+0.15 \cos (t)) y_{1}(t-0.25)+(3.5+0.1 \cos (t)) y_{2}(t-0.25)+2+0.15 \cos (t)}\right] .
\end{aligned}
$$

Hence,

$$
\begin{aligned}
& M_{1} \approx 1.1795, M_{2} \approx 1.0260, M_{3} \approx 1.0141, m_{1} \approx 0.2403, m_{2} \approx 0.2440, m_{3} \approx 0.2135 \\
& \Gamma_{1}^{L} \approx 9.1129, \Gamma_{2}^{L} \approx 9.4947, \Gamma_{3}^{L} \approx 12.1427, A_{1}^{M} \approx 0.3333, A_{2}^{M} \approx 0.4882, A_{3}^{M} \approx 0.4985 \\
& A_{4}^{M} \approx 0.5679, A_{5}^{M} \approx 0.5591, A_{6}^{M} \approx 0.3128, A_{7}^{M} \approx 0.5367, A_{8}^{M} \approx 0.4542, A_{9}^{M} \approx 0.4668 \\
& A_{10}^{M} \approx 0.4595, A_{11}^{M} \approx 0.2479, A_{12}^{M} \approx 0.4964, A_{13}^{M} \approx 0.4258, A_{14}^{M} \approx 0.4268, A_{15}^{M} \approx 0.4209
\end{aligned}
$$

and

$$
B_{1} \approx 1.6939, B_{2} \approx 1.5576, B_{3} \approx 1.3613, \quad B=\min \left\{B_{i}(i=1,2,3)\right\}=1.3613
$$

It is easy to show that system (4.1) satisfies the conditions of Theorem 3.1, Theorem 3.4, Corollary 3.3 and Corollary 3.5. Hence, system (4.1) is permanent, globally attractive and has a globally attractive positive periodic solution. The permanence, periodic solution and globally attractive of system (4.1) are shown in Figure 4.1. 

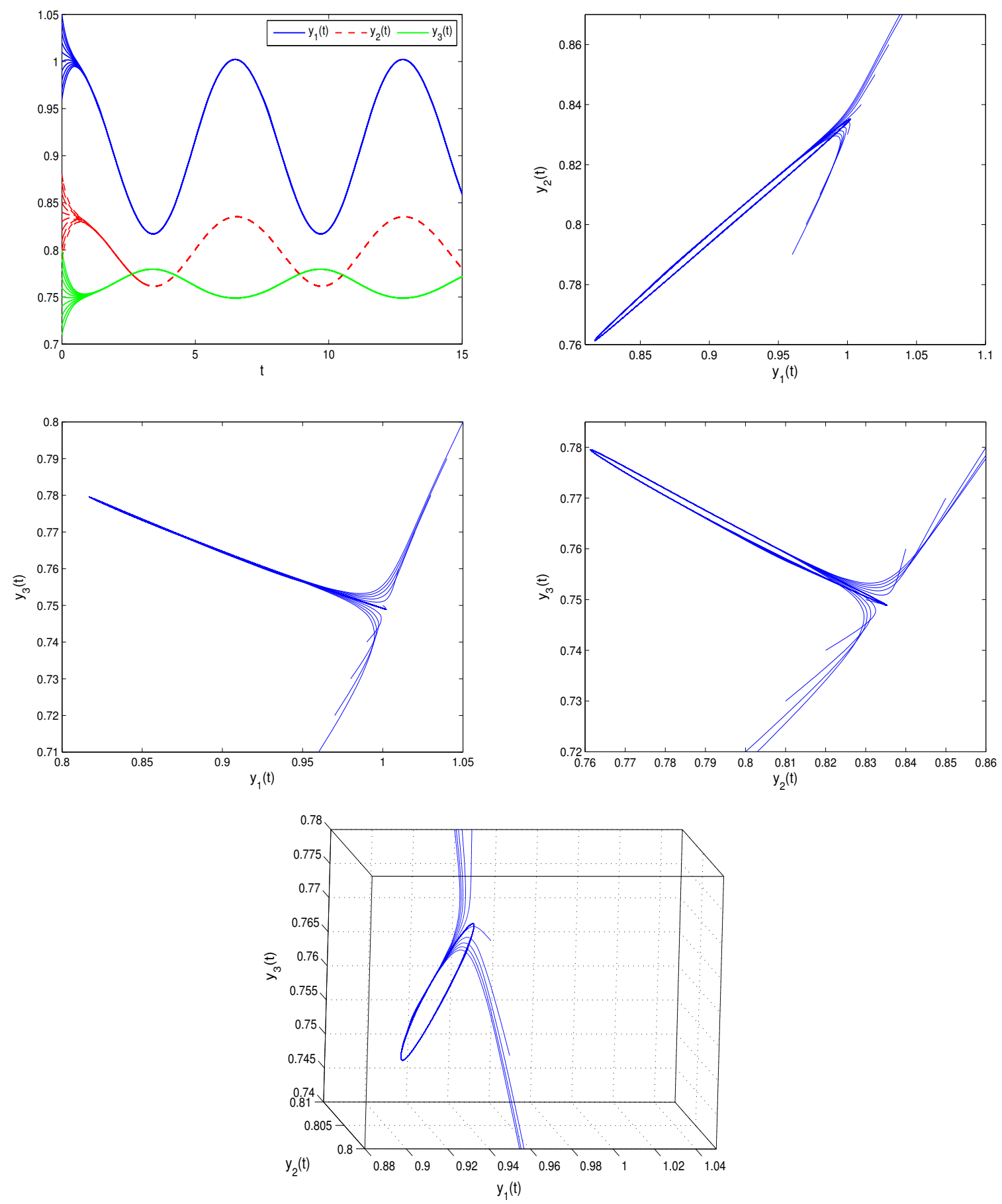

Fig 4.1. The numerical solutions of system (4.1) with different initial conditions.

From the Fig 4.1 we can see that system (4.1) is permanent and has a globally attractive positive periodic solution.

\section{THE CONCLUSION}

In this paper, based on the previous works [7]-[15], one class of non-autonomous three species Lotka-Volterra cooperative system with ratio-dependent functional responses and time 
delays was proposed. With the aid of the comparison method and the construction of Lyapunov functions, we obtained the sufficient conditions on the permanence, existence of positive periodic solutions, and the global attractivity of system (1.4). In addition, some numerical solutions were given to show the feasibility of our results. Moreover, from the conditions of Theorem 3.1 and Theorem 3.4, we have that the conditions of the permanence and global attractivity are not restrictive.

\section{Acknowledgements}

This project is supported by the National Natural Science Foundation of Xinjiang (No. 2021D01 C067), the fund of the Guangdong Provincial Key Laboratory of Computational Science and Material Design (No. 2019B030301001) and National Natural Science Foundation of China (Grant No. 11662020,11861063).

\section{REFERENCES}

[1] S. Hsu, T. Hwang, Y. Kuang, Rich dynamics of a ratio-dependent one prey two predators model, J. Math/ Biolo. 43 (2001), 377-396.

[2] S. Chen, F. Wang, T. Young, Positive periodic solution of two-species ratio-dependent predator-prey system with time delay in two-patch environment, Appl. Math. Comput. 150 (2004),737-748.

[3] D. Xiao, W. Li , M. Han, Dynamics in a ratio-dependent predator-prey model with predator harvesting, J. Math. Anal. Appl.s 324 (2006), 14-29.

[4] F. Chen, M. You, Permanence, extinction and periodic solution of the predator-prey system with BeddingtonDeAngelis functional response and stage structure for prey, Nonlinear Anal. 9 (2008),207-221.

[5] Z. Li, L. Chen, J. Huang, Permanence and periodicity of a delayed ratio-dependent predator-prey model with Holling type functional response and stage structure, J. Comput. Appl. Math. 233 (2009),173-187.

[6] A Muhammadhaji ,Z Teng, Permanence and extinction analysis for a periodic competing predator-prey system with stage structure, Int. J. Dyn. Control 5 (2017), 858-871.

[7] R.M. May, Theoretical Ecology, Principle and Applications, Sounders, Philadelphia, PA, 1976.

[8] L. Chen, Models and Research Methods of Mathematical Ecology, Science Press, Beijing, 1988.

[9] J. Cui, Global asymptotic stability in n-species cooperative system with time delays, Sys. Sci. Math. Sci. 7 (1994), 45-48.

[10] J. Zhang, L. Chen, Permanence and global stability for a two-species cooperative system with time delays in a two-patch environment, Comput. Math. Applic. 32 (1996), 101-108.

[11] A Muhammadhaji ,Z. Teng, X. Abdurahman, Permanence and extinction analysis for a delayed ratiodependent cooperative system with stage structure, Afrika Matematika 25 (2014), 897-909.

[12] F. Chen, J. Yang, L. Chen, X. Xie, On a mutualism model with feedback controls, Appl. Math. Comput. 214 (2009), 581-587.

[13] L. Chen, X. Xie, Permanence of an N-species cooperation system with continuous time delays and feedback controls, Nonlinear Anal. 12 (2011), 34-38.

[14] L. Yang, X. Xie, F Chen, Y. Xue, Permanence of the periodic predator-prey-mutualist system, Adv. Difference Equ. 2015 (2015), 331.

[15] L. Zhao, B. Qin, F Chen, Permanence and global stability of a May cooperative system with strong and weak cooperative partners, Adv. Difference Equ. 172 (2018), 1-13.

[16] A. Muhammadhaji, Z. Teng, M. Rahim, Dynamical behavior for a class of delayed competitive-mutualism systems, Differ. Equ. Dyn. Syst. 23 (2015), 281-301.

[17] Z. Teng, L. Chen, The positive periodic slotions in periodic Kolmogorov type systems with delays, Acta. Math. Appl. Sin. 22 (1999), 446-456. 